\title{
Comunidade de morcegos (Mammalia, Chiroptera) no Refúgio de Vida Silvestre Mata do Junco, Sergipe, nordeste do Brasil
}

\author{
Daniela de Vasconcelos Brito $^{1}$ \& Adriana Bocchiglieri ${ }^{1,2,3}$ \\ ${ }^{I}$ Núcleo de Pós-graduação em Ecologia e Conservação, Cidade Universitária Prof. José Aloísio de \\ Campos, Universidade Federal de Sergipe - UFS, CEP 49100-000, São Cristóvão, SE, Brasil. \\ http://www.pos.ufs.br/ecologial \\ ${ }^{2}$ Departamento de Biologia, Cidade Universitária Prof. José Aloísio de Campos, \\ Universidade Federal de Sergipe - UFS, CEP 49100-000, São Cristóvão, SE, Brasil. http://www.dbi.ufs.br \\ ${ }^{3}$ Autor para correspondência: Adriana Bocchiglieri, e-mail: adriblue@hotmail.com
}

BRITO, D.V. \& BOCCHIGLIERI, A. Bats community (Mammalia, Chiroptera) in Refúgio de Vida Silvestre Mata do Junco, Sergipe, northeastern Brazil. Biota Neotrop. 12(3): http://www.biotaneotropica.org.br/v12n3/ en/abstract?inventory+bn02112032012

\begin{abstract}
The order Chiroptera is the second in mammal species richness in Brazil and the Atlantic Forest is the biome with the best knowledge status for this group. In Sergipe, studies focused on bats are still scarce, being necessary to increase the research on such important taxon. This work aimed to conduct a bat inventory in the Refúgio de Vida Silvestre Mata do Junco (RVSMJ), which is the second largest reserve of Atlantic Forest in the state of Sergipe. Samples were obtained during two nights per month from February 2011 to February 2012, except in June. With a sampling effort of 21,168 $\mathrm{m}^{2}$.h, we recorded 189 individuals of two families and 14 species. Among these, Artibeus planirostris, Chiroderma doriae, Myotis nigricans, Phyllostomus discolor, Trachops cirrhosus and Trinycteris nicefori are new occurrences for the location, being T. nicefori also new record for the state. Phyllostomidae was the richest and most abundant family, and A. lituratus $(\mathrm{N}=67)$, Carollia perspicillata $(\mathrm{N}=45)$, and Dermanura cinerea $(\mathrm{N}=45)$ were the most abundant species. These three species accounted for $83.0 \%$ of all captured individuals, and were characterized as generalists in habitat use and diet. With respect to feeding guilds, most of the captured species are frugivores (57.1\%), probably due to the methodology and existence of agricultural areas present in RVSMJ. The new records here assigned, along with the parameters obtained for richness, abundance and feeding guild, will increase our knowledge on the bats of Sergipe and can be used as a basis for conservation strategies and management at the studied site location.
\end{abstract}

Keywords: Atlantic Forest, richness, trophic guild, abundance, Trinycteris nicefori.

BRITO, D.V. \& BOCCHIGLIERI, A. Comunidade de morcegos (Mammalia, Chiroptera) no Refúgio de Vida Silvestre Mata do Junco, Sergipe, nordeste do Brasil. Biota Neotrop. 12(3): http://www.biotaneotropica.org. br/v12n3/pt/abstract?inventory+bn02112032012

Resumo: A ordem Chiroptera é a segunda maior em riqueza de mamíferos no Brasil e a Mata Atlântica é o bioma com o melhor estado de conhecimento para esse grupo. Em Sergipe, estudos sobre quirópteros ainda são escassos, sendo necessário ampliar as pesquisas com esses animais. O presente trabalho teve como objetivo realizar o inventário de morcegos do Refúgio de Vida Silvestre Mata do Junco (RVSMJ), segunda maior reserva de Mata Atlântica do estado de Sergipe. As coletas foram realizadas durante duas noites por mês, entre fevereiro de 2011 a fevereiro de 2012, exceto junho. Com um esforço de captura de $21.168 \mathrm{~m}^{2}$.h foram registrados 189 indivíduos de duas famílias e 14 espécies. Destas, Artibeus planirostris, Chiroderma doriae, Myotis nigricans, Phyllostomus discolor, Trachops cirrhosus e Trinycteris nicefori correspondem a novas ocorrências para a localidade, sendo T. nicefori novo registro para o estado. Phyllostomidae foi a família com maior riqueza e abundância e A. lituratus $(\mathrm{N}=67)$, Carollia perspicillata $(\mathrm{N}=45)$ e Dermanura cinerea $(\mathrm{N}=45)$ foram as espécies mais abundantes. Essas três espécies juntas representam 83,0\% dos indivíduos capturados; sendo caracterizadas como generalistas no uso do habitat e dieta. Com relação às guildas alimentares, a maioria das espécies é frugívora $(57,1 \%)$, devido a metodologia e existência de áreas agrícolas presentes no RVSMJ. O registro de novas espécies para a localidade, juntamente com os parâmetros obtidos de riqueza, abundância e guilda alimentar, contribuem para o aumento do conhecimento dos quirópteros em Sergipe e podem servir de base para estratégias de conservação e manejo dessa área.

Palavras-chave: Mata Atlântica, riqueza, guilda trófica, abundância, Trinycteris nicefori. 


\section{Introdução}

A Mata Atlântica apresenta uma rica fauna e flora, representadas por 22.000 espécies de angiospermas e 2.039 de vertebrados, dos quais são conhecidas 270 espécies de mamíferos, sendo 73 delas endêmicas (Campanili \& Schaffer 2010). Nesse sentido, este bioma é considerado o segundo maior em riqueza e taxa de endemismo para a mastofauna brasileira (Reis et al. 2011). Apesar de originalmente ocupar uma área equivalente a $15 \%$ do território nacional (Brasil 2002), atualmente a Mata Atlântica apresenta-se com $88,27 \%$ de sua área reduzida e fragmentada (Ribeiro et al. 2009). Esses processos de alteração e devastação da paisagem resultaram em poucas áreas conservadas e grandes o suficiente para garantir a manutenção de sua biodiversidade, o que faz desse bioma um dos mais ameaçados do mundo (Campanili \& Schaffer 2010) e um dos 34 hotspots globais (Mittermeier et al. 2005).

Além de reduzidos, os remanescentes florestais desse bioma estão fragmentados (Campanili \& Schaffer 2010), sendo esta uma das maiores ameaças a mastofauna brasileira (Costa et al. 2005). Estudos na Mata Atlântica envolvendo mamíferos revelaram que esses animais têm seus padrões de diversidade, abundância e riqueza influenciados negativamente pelos efeitos da fragmentação (e.g. Vieira et al. 2003, Pardini 2004, Pardini et al. 2005, 2009). Particularmente para os quirópteros, uma variação na disponibilidade dos recursos em ambientes fragmentados pode alterar a estrutura da comunidade desses animais (Faria 2006, Schulze et al. 2000).

A ordem Chiroptera é a segunda maior em riqueza de mamíferos no Brasil, com 172 espécies distribuídas em nove famílias (Reis et al. 2011), sendo a Mata Atlântica o bioma com melhor estado de conhecimento para os representantes desse grupo (Bernard et al. 2011). Atualmente as espécies Lonchophylla bokermanni, Lasiurus ebenus, Platyrrhinus recifinus e Myotis ruber encontram-se ameaçadas; sendo que as duas últimas apresentam distribuição para a região Nordeste (Chiarello et al. 2008). Nessa região, a Mata Atlântica cobria aproximadamente $29 \%$ do território, restando hoje um pouco mais de $2 \%$ distribuídos em pequenos fragmentos remanescentes (Tabarelli et al. 2011). No menor estado brasileiro, Sergipe, esse bioma ocupava toda a faixa litorânea (Landim \& Siqueira 2001). Sua área natural foi, entretanto, bastante devastada, principalmente para fins agropastoris (Santos \& Santos 2006), restando pouco mais de 9\% da sua cobertura florestal original (Fundação... \& Instituto... 2011).

Trabalhos com quirópteros realizados em Mata Atlântica no estado tratam sobre a estrutura da comunidade (Mikalauskas 2005, Rocha et al. 2010), padrão de atividade (Mikalauskas et al. 2006a), uso do habitat (Mikalauskas 2005) e novas ocorrências de espécies (Mikalauskas et al. 2006b, 2011, Feijó \& Nunes 2010, Rocha et al. 2010, 2011a, b). Mesmo assim, estudos ecológicos sobre os quirópteros em Sergipe ainda são escassos (Rocha et al. 2010).

Considerando a escassez sobre o conhecimento da ocorrência e estrutura da quiropterofauna sergipana, é importante ampliar as pesquisas com esse grupo no estado. Neste contexto, a caracterização das comunidades de morcegos é fundamental para a obtenção de dados ecológicos que servirão de base para futuros estudos, além da adoção de estratégias de conservação desses animais e dos remanescentes florestais que os abrigam. Dessa forma, o presente trabalho tem por objetivo caracterizar a comunidade de morcegos do Refúgio de Vida Silvestre Mata do Junco em Sergipe.

\section{Material e Métodos}

O Refúgio de Vida Silvestre Mata do Junco - RVSMJ (1046’ S e $\left.37^{\circ} 01^{\prime} \mathrm{W}\right)$ está localizado ao leste do estado de Sergipe, no município de Capela (Figura 1a). Esta unidade de conservação é considerada a segunda maior reserva de Mata Atlântica do estado, com 1520 hectares
(Figura 1b) (Santos et al. 2007). O RVSMJ é caracterizado como um remanescente florestal subdecidual (Santos et al. 2007), constituído basicamente por três estratos arbóreos distintos: o sub-bosque, caracterizado por espécies com folhas largas; o subdossel, composto por arvoretas, lianas e trepadeiras; e o dossel, formado por árvores acima de 15 metros de altura (Dantas et al. 2007).

No presente estudo foram selecionados dois sítios de coleta no fragmento maior que compõe o RVSMJ com o propósito de uma melhor caracterização da fauna de morcegos na área. O primeiro sítio corresponde à uma mancha florestal próxima a sede da unidade de conservação, caracterizada por uma vegetação secundária baixa, com a presença de clareiras e um sub-bosque denso, formado por um emaranhado de cipós e arbustos (observação pessoal). O segundo sítio corresponde ao fragmento florestal associado à nascente do riacho Lagartixo, próximo a Estação de Captação de Água e Esgoto (SAAE), caracterizado por uma vegetação com árvores altas (cerca de 15-18 m de altura) e que apresenta-se preservada (Ferreira et al. 2007).

As campanhas de campo foram realizadas entre fevereiro de 2011 e fevereiro de 2012, com exceção do mês de junho, alternadamente em cada sítio. As coletas ocorreram em duas noites consecutivas por mês utilizando-se sete redes de neblina $(7 \times 3 \mathrm{~m})$ que permaneceram abertas das $18 \mathrm{~h} 00$ até $24 \mathrm{~h} 00$, sendo revisadas em intervalos de meia hora. Para a identificação dos espécimes capturados utilizou-se informações disponíveis em Gardner (2008), Peracchi et al. (2010, 2011), Reis et al. (2007) e Vizotto \& Taddei (1973), a partir de caracteres morfológicos, externos e dentários.

Os animais foram marcados na asa esquerda com anilhas plásticas numeradas e depois soltos no mesmo local de captura. Para confirmação da identificação e registro da espécie na localidade amostrada, exemplares foram sacrificados, fixados com formol a $10 \%$ e conservados em álcool $70 \%$, sendo posteriormente depositados na Coleção de Mamíferos da Universidade Federal de Sergipe (CMUFS), segundo Licença de Pesquisa e Coleta número 2011.05.0108/00113011 da SEMARH - SE (Apêndice 1).

Para a identificação de novos registros para a localidade foram utilizados os dados pretéritos de Rocha et al. (2007) e Mikalauskas et al. (2011). Todas as espécies tiveram a abundância contabilizada e foram classificadas de acordo com seus hábitos alimentares predominantes, a partir de informações disponíveis em Peracchi et al. (2010, 2011) e Reis et al. (2007). O esforço de captura foi calculado segundo Straube \& Bianconi (2002). Para avaliar se este esforço foi satisfatório e realizar uma estimativa da riqueza na área, foram construídas curvas médias de acumulação de espécies com o aumento do esforço amostral, aleatorizadas 10.000 vezes, através do programa EstimateSWin 8.2 (Colwell 2011). Essas curvas foram obtidas para dois estimadores não-paramétricos (Jackknife1 e Jackknife2) que se baseiam na ocorrência de espécies raras e no número de amostras (Santos 2003), sendo escolhido o que apresentou o menor desvio padrão.

\section{Resultados}

Com um esforço amostral de $21.168 \mathrm{~m}^{2}$.h foram capturados 189 indivíduos, com cinco recapturas, pertencentes a duas famílias, 12 gêneros e 14 espécies (Tabela 1). Destas, seis representam novas ocorrências para o RVSMJ: Artibeus planirostris (Spix, 1823), Chiroderma doriae Thomas, 1891, Myotis nigricans (Schinz, 1821), Phyllostomus discolor (Wagner, 1843), Trachops cirrhosus (Spix, 1823) e Trinycteris nicefori Sanborn, 1949, totalizando 17 espécies registradas nessa localidade (Tabela 1 ).

Através das curvas médias de acumulação obteve-se uma estimativa de riqueza de $18,58 \pm 2,86$ espécies por meio do estimador não paramétrico Jackknife 1, sendo que a curva não atingiu a assíntota 
(Figura 2). Estes resultados indicam que a riqueza observada ao longo do estudo representa 75,3\% das espécies esperadas para o RVSMJ.

A família Phyllostomidae (Figura 3) foi a mais rica e a mais abundante, com $92,8 \%$ das espécies registradas e aproximadamente $98,4 \%$ das capturas, enquanto a família Vespertilionidae apresentou três indivíduos capturados de uma única espécie. Dos filostomídeos, a subfamília Stenodermatinae foi a mais representativa, com 127 indivíduos e sete espécies capturadas.

As espécies mais abundantes foram Artibeus lituratus com 67 indivíduos (35,4\%) Carollia perspicillata com $45(23,8 \%)$ e Dermanura cinerea com 45 (23,8\%), representando juntas $83,0 \%$ dos indivíduos capturados (Figura 4). Duas espécies (Tonatia saurophila e Trachops cirrhosus) tiveram somente um indivíduo capturado (Figura 4).

Com relação aos hábitos alimentares das espécies obtidas nesse estudo, $57,1 \%$ delas são frugívoras, seguida pelas insetívoras com $21,4 \%$ (Tabela 1). Os demais registros foram de espécies nectarívoras, onívoras e carnívoras, com $7,1 \%$ cada (Tabela 1 ).

\section{Discussão}

Na comunidade de morcegos do RVSMJ eram conhecidas, até o momento, 11 espécies (Rocha et al. 2007, Mikalauskas et al. 2011), das quais oito $(72,7 \%)$ foram capturadas no presente estudo. $\mathrm{O}$ registro pretérito de Tonatia bidens nessa localidade como a primeira ocorrência para Sergipe, apresentado por Rocha et al. (2007), na verdade corresponde a espécie Tonatia saurophila (P.A. Rocha, comunicação pessoal), também coletada nesse trabalho. Houve um acréscimo de aproximadamente 54,5\% na riqueza do RVSMJ, resultando em 17 espécies. Contudo, a riqueza observada foi menor do que a esperada pela curva de acumulação que não atingiu a assíntota, indicando que o esforço amostral foi incipiente e que novas espécies ainda podem ser amostradas na localidade.

Dos seis novos registros obtidos para a área, um é novo para o estado. A captura de Trinycteris nicefori constitui a primeira ocorrência em Sergipe e a ampliação de sua distribuição no Nordeste. Essa espécie possui registro para Mata Atlântica e Floresta Amazônica, podendo ser encontrada nos estados do Acre, Amazonas, Amapá, Bahia, Espírito Santo, Mato Grosso, Pará, Roraima e Tocantins (Peracchi et al. 2010); sendo o registro na Bahia o primeiro a ser obtido para o Nordeste (Faria et al. 2006).

Estudos com morcegos realizados em áreas de Mata Atlântica têm apresentado uma riqueza que varia entre 14-15 espécies (e.g Miretzki \& Margarido 1999, Baptista \& Mello 2001, Andrade et al. 2010) a mais de 25-40 espécies (e.g. Muller \& Reis 1992, Esbérard 2003, Faria \& Baumgartem 2007, Silva et al. 2010). Além das diferenças no esforço amostral, essa variação na riqueza pode estar associada ao número de áreas amostradas. Assim, os trabalhos citados acima que apresentaram uma menor riqueza, como o do RVSMJ, tiveram poucos pontos amostrados localmente, não compreendendo uma amostragem que refletisse a totalidade da área estudada.

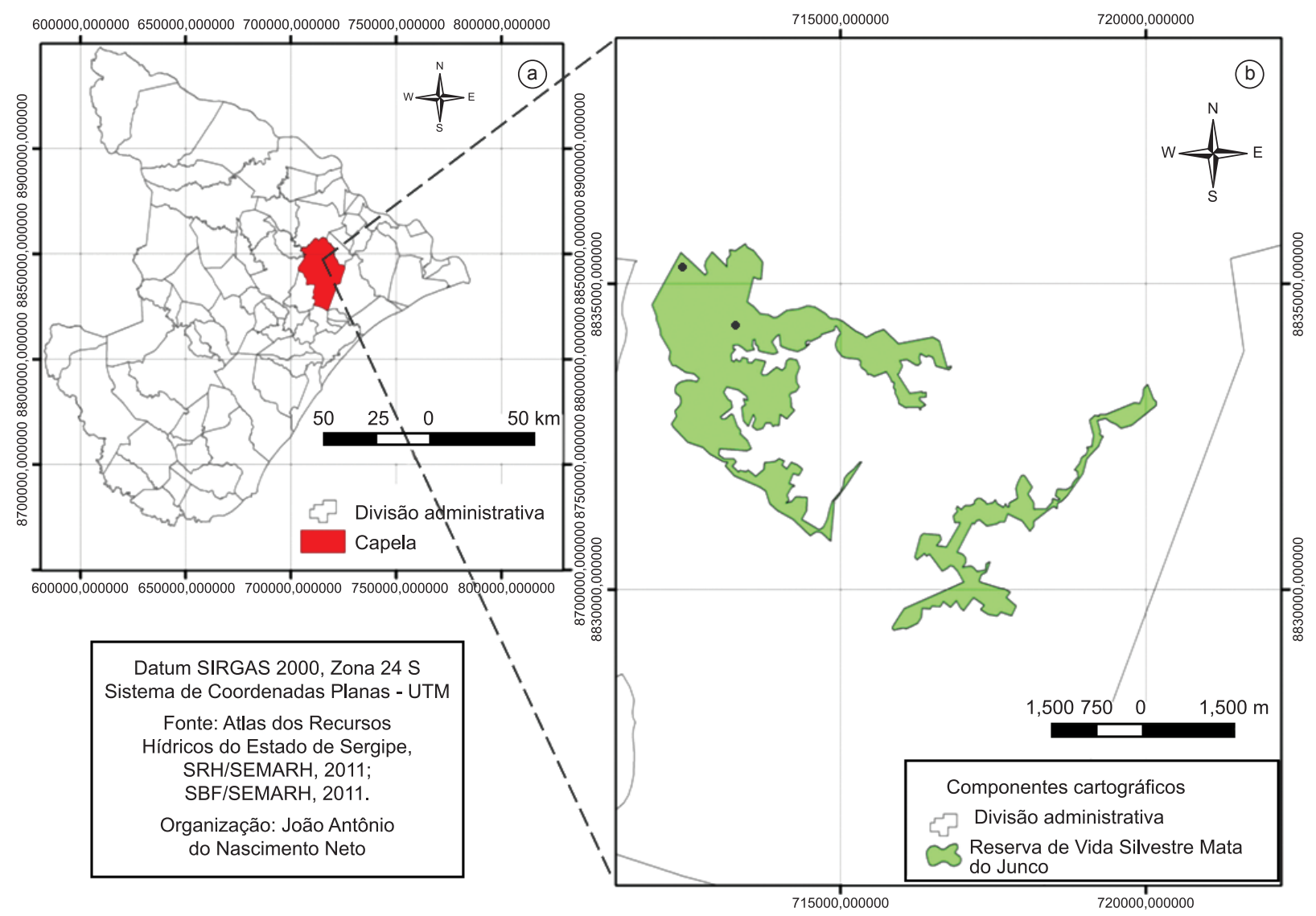

Figura 1. Mapa do estado de Sergipe indicando a localização do município de Capela (a) e a localização do Refúgio de Vida Silvestre Mata do Junco e sítios de coleta (b).

Figure 1. Map of the state of Sergipe indicating the location of the Capela city (a) and location of the Refúgio de Vida Silvestre Mata do Junco and sampling sites (b). 
Tabela 1. Relação das espécies de morcegos registradas no Refúgio de Vida Silvestre Mata Junco, estado de Sergipe, e das espécies capturadas no presente estudo, com suas respectivas guildas tróficas.

Table 1. List of bat species recorded in the Refúgio de Vida Silvestre Mata do Junco, state of Sergipe, and species captured in this study with their respective feeding guilds.

\begin{tabular}{|c|c|c|c|}
\hline Espécies & RVSMJ $^{1}$ & Presente estudo & Guilda trófica \\
\hline \multicolumn{4}{|l|}{ Família Phyllostomidae } \\
\hline \multicolumn{4}{|l|}{ Subfamília Desmodontinae } \\
\hline Desmodus rotundus (É. Geoffroy, 1810) & $\mathrm{X}$ & & hematófago \\
\hline \multicolumn{4}{|l|}{ Subfamília Phyllostominae } \\
\hline Phyllostomus discolor (Wagner, 1843) & & $\mathrm{X}$ & onívoro \\
\hline Phyllostomus hastatus (Pallas, 1767) & $\mathrm{X}$ & & onívoro \\
\hline Tonatia saurophila Koopman e Williams, 1951 & $\mathrm{X}$ & $\mathrm{X}$ & insetívoro \\
\hline Trachops cirrhosus (Spix, 1823) & & $\mathrm{X}$ & carnívoro \\
\hline Trinycteris nicefori Sanborn, $1949 *$ & & $\mathrm{X}$ & insetívoro \\
\hline \multicolumn{4}{|l|}{ Subfamília Stenodermatinae } \\
\hline Artibeus fimbriatus Gray, 1838 & $\mathrm{X}$ & $\mathrm{X}$ & frugívoro \\
\hline Artibeus lituratus (Olfers, 1818) & $\mathrm{X}$ & $\mathrm{X}$ & frugívoro \\
\hline Artibeus planirostris (Spix, 1823) & & $\mathrm{X}$ & frugívoro \\
\hline Chiroderma doriae Thomas, 1891 & & $\mathrm{X}$ & frugívoro \\
\hline Dermanura cinerea Gervais, 1856 & $\mathrm{X}$ & $\mathrm{X}$ & frugívoro \\
\hline Platyrrhinus lineatus (É. Geoffroy, 1810) & $\mathrm{X}$ & $\mathrm{X}$ & frugívoro \\
\hline Sturnira lilium (É. Geoffroy, 1810) & $\mathrm{X}$ & $\mathrm{X}$ & frugívoro \\
\hline \multicolumn{4}{|l|}{ Subfamília Carolliinae } \\
\hline Carollia perspicillata (Linnaeus, 1758) & $\mathrm{X}$ & $\mathrm{X}$ & frugívoro \\
\hline \multicolumn{4}{|l|}{ Subfamília Glossophaginae } \\
\hline Glossophaga soricina (Pallas, 1766) & $\mathrm{X}$ & $\mathrm{X}$ & nectarívoro \\
\hline \multicolumn{4}{|l|}{ Família Vespertilionidae } \\
\hline Myotis nigricans (Schinz, 1821) & & $\mathrm{X}$ & insetívoro \\
\hline Rhogeessa hussoni Genoways e Baker, 1996 & $\mathrm{X}$ & & insetívoro \\
\hline
\end{tabular}

*Novo registro para Sergipe. ${ }^{1}$ Rocha et al. (2007) e Mikalauskas et al. (2011).

*New record for Sergipe. ${ }^{1}$ Rocha et al. (2007) and Mikalauskas et al. (2011).

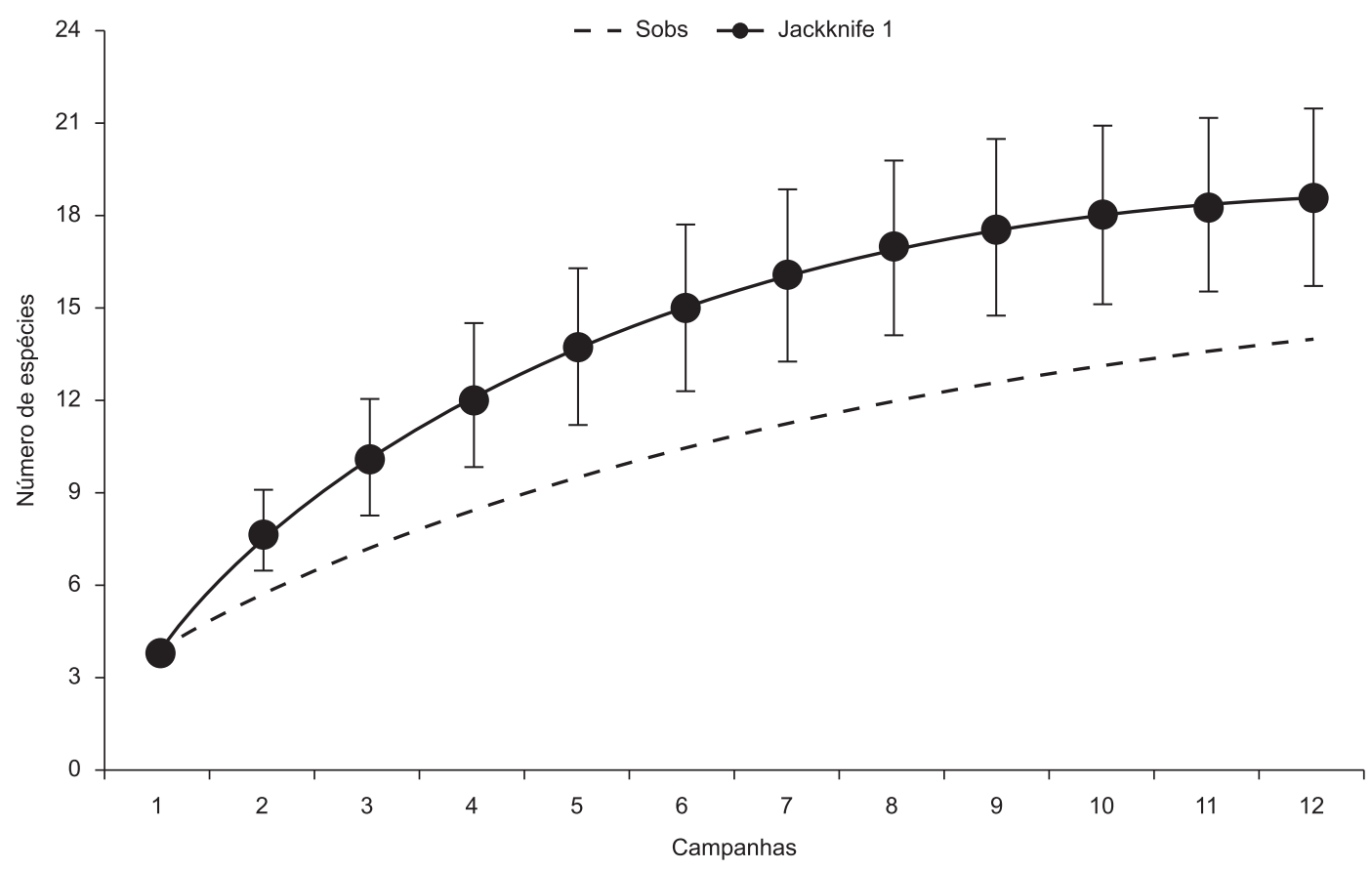

Figura 2. Curvas de acumulação de espécies de morcegos observada ( - - - ) e estimada (- - ) pelo estimador não paramétrico Jackknife 1 em campanhas realizadas entre fevereiro de 2011 a fevereiro de 2012 no Refúgio de Vida Silvestre Mata do Junco/Sergipe. As linhas verticais representam o desvio padrão.

Figure 2. Accumulation curves of bats species observed ( - - - ) and estimated (-) by nonparametric Jackknife 1 estimator in a campaign realized between February 2011 and February 2012 in Refúgio de Vida Silvestre Mata do Junco/Sergipe. Vertical lines represents the standard deviation 

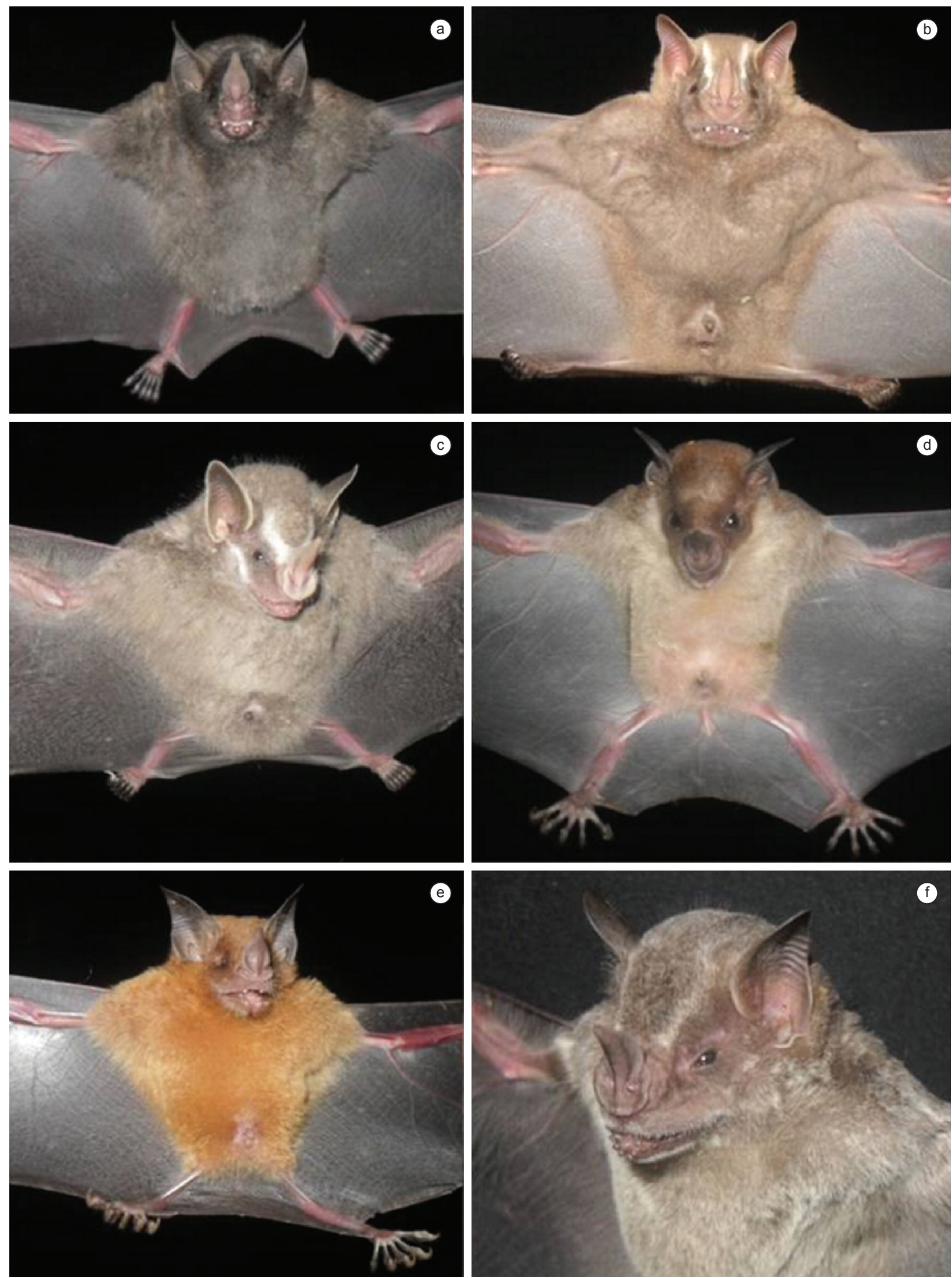

Figura 3. Representantes de morcegos da família Phyllostomidae capturados no Refúgio de Vida Silvestre Mata do Junco/Sergipe. a) Carollia perspicillata; b) Artibeus lituratus; c) Dermanura cinerea; d) Phyllostomus discolor; e) Trinycteris nicefori e f) Artibeus planirostris.

Figure 3. Representatives of the family Phyllostomidae bats captured in Refúgio de Vida Silvestre Mata do Junco/Sergipe. a) Carollia perspicillata; b) Artibeus lituratus; c) Dermanura cinerea; d) Phyllostomus discolor; e) Trinycteris nicefori and f) Artibeus planirostris. 


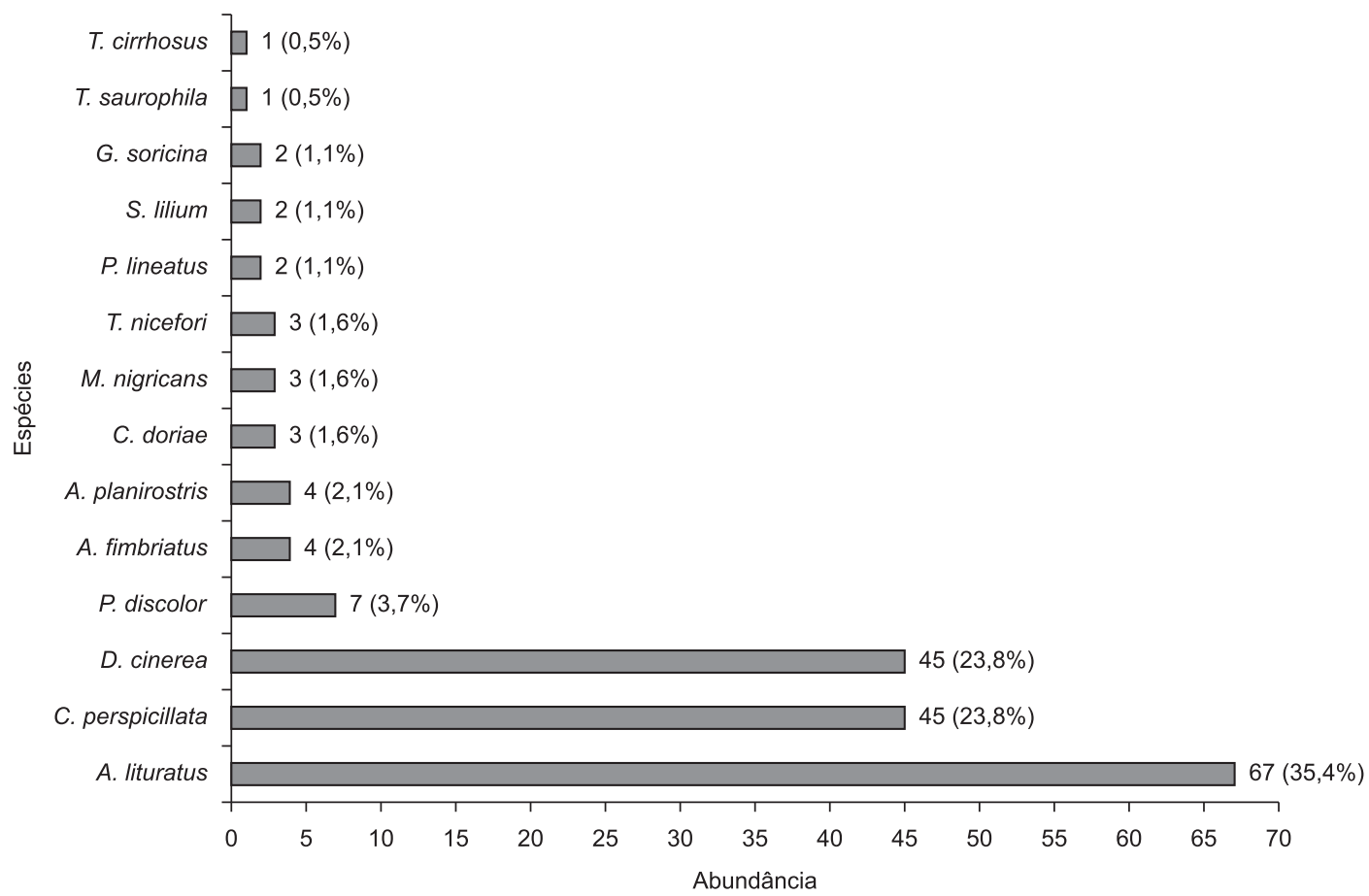

Figura 4. Abundância absoluta e relativa (em \%) das espécies de morcegos registradas no Refúgio de Vida Silvestre Mata do Junco/Sergipe entre fevereiro de 2011 a fevereiro de 2012.

Figure 4. Absolute and relative abundance (in \%) of bats species recorded in Refúgio de Vida Silvestre Mata Junco/Sergipe from February 2011 to February 2012.

Outro fator que deve ser considerado na obtenção de um inventário representativo da quiropterofauna local é a limitação metodológica, sendo recomendada a utilização de diferentes métodos de amostragem (Bergallo et al. 2003). No caso do uso de redes de neblina, há uma maior captura de filostomídeos (e.g. Trajano 1984, Pedro \& Taddei 1998, Ortêncio-Filho \& Reis 2009). Portanto, a elevada representatividade desses morcegos nos parâmetros obtidos no RVSMJ já era esperada, em parte devido o uso de exclusivo desse método de coleta. Também deve ser considerada que essa é a família com maior riqueza no Brasil, com aproximadamente 53\% das espécies registradas no país (Peracchi et al. 2011).

A maior riqueza e abundância observada na subfamília Stenodermatinae também é justificada pela metodologia adotada, uma vez que esses animais são caracterizados como frugívoros de dossel e sub-bosque (Rex et al. 2008); sendo que morcegos com voo baixo são mais facilmente capturados pela rede de neblina (Pedro \& Taddei 1998). Por outro lado, a pouca representatividade de vespertilionídeos pode ser explicada pela habilidade de alguns quirópteros em evitar as redes (Kunz \& Kurta 1988), como é o caso de algumas espécies insetívoras, que conseguem detectá-las com facilidade (Trajano 1984).

Três espécies representaram juntas $83,0 \%$ dos indivíduos capturados, sendo este resultado um padrão comum em comunidades neotropicais, onde há um alto número de espécies raras e poucas dominantes (Trajano 1984). A elevada abundância observada para Artibeus lituratus e Carollia perspicillata provavelmente está relacionada com a estrutura ambiental do RVSMJ, a qual apresenta locais com intensa ação antrópica (Santos et al. 2007). Essas duas espécies são consideradas indicadoras de habitats alterados (Muller \& Reis 1992, Reis et al. 2003), visto que se adaptam bem a esses ambientes e são menos exigentes na seleção dos alimentos. De forma semelhante, o número de indivíduos observado para Dermanura cinerea na área possivelmente está associado à utilização, por esta espécie, de diversos habitats como florestas primárias, secundárias e remanescentes (Zortéa 2007); ambientes também encontrados no RVSMJ.

Com relação à distribuição das guildas alimentares, os frugívoros foram os que apresentaram maior número de indivíduos, sendo esse resultado reflexo do fato das três espécies mais abundantes obtidas nesse trabalho possuírem esse hábito alimentar. Esse grupo tende a ser bastante amostrado com o uso de redes de neblina (Arita 1993, Bergallo et al. 2003), e a existência de áreas agrícolas inseridas no RVSMJ também pode ter favorecido sua elevada representatividade, pois esses ambientes podem servir de locais de alimentação para muitas espécies (Trajano 1984, Mikalauskas 2005).

O registro de novas espécies para o RVSMJ, juntamente com os parâmetros obtidos de riqueza, abundância e guilda trófica, contribuem para o aumento do conhecimento dos quirópteros em Sergipe, principalmente considerando os poucos trabalhos sobre a caracterização da comunidade desses animais no estado (e. g. Mikalauskas 2005, Rocha et al. 2010). Além disso, observou-se que novas espécies podem ser encontradas na localidade, sendo necessária a ampliação e diversificação dos locais amostrados, utilização de outros métodos de coleta e a realização de um maior esforço amostral na área.

Estudos como o realizado no RVSMJ reforçam a importância das unidades de conservação na manutenção da diversidade local e servem de diagnóstico da qualidade ambiental dessas áreas. Nesse sentido, compreender como as espécies de morcegos estão distribuídas e suas interações com o ambiente e os outros organismos, pode servir de base para estratégias de conservação e manejo da localidade estudada.

\section{Agradecimentos}

Ao Laboratório de Mastozoologia da UFS, em especial a Daiany S. Reis, Déborah M. Melo, Lúcia G.F. Mello e Marianna N. Oliveira 
pela contribuição na coleta dos dados; a Patrício A. Rocha e Jefferson S. Mikalauskas pelas informações e auxílio na identificação dos animais; aos funcionários do Refúgio de Vida Silvestre Mata do Junco e a SEMARH pelo apoio logístico.

\section{Referências Bibliográficas}

ANDRADE, L.P., MELO, R.S., SILVA, R.R., OLIVEIRA, P.J.A., OLIVEIRA, J.C.T., SILVA, E.M.V.G., SILVA FILHO, T.P. \& SILVA, L.A.M. 2010. Quiropterofauna da Estação Ecológica de Caetés, Paulista, Pernambuco, Brasil: atualização do inventário 10 anos depois. Chirop. Neotrop. Supl. 16(1):75-77.

ARITA, H.T. 1993. Rarity in Neotropical Bats: correlations with phylogeny, diet, and body mass. Ecol. Appl. 3(3):506-517. http://dx.doi. org/10.2307/1941919

BAPTISTA, M. \& MELLO, M. 2001. Preliminary inventory of the bat species of the Poço das Antas Biological Reserve, RJ. Chirop. Neotrop. 7(1-2):133-135.

BERGALLO, H.G., ESBÉRARD, C.E.L., MELLO, M.A.R., LINS, V., MANGOLIN, R., MELO, G.G.S. \& BAPTISTA, M. 2003. Bat species richness in Atlantic Forest: what is the minimum sampling effort? Biotropica 35(2):278-288. http://dx.doi.org/10.1111/j.1744-7429.2003. tb00286.x

BERNARD, E., AGUIAR, L.M.S. \& MACHADO, R.B. 2011. Discovering the brazilian bat fauna: a task for two centuries? Mammal Rev. 41(1):23-39. http://dx.doi.org/10.1111/j.1365-2907.2010.00164.x

BRASIL. Ministério do Meio Ambiente - MMA. 2002. Biodiversidade brasileira: avaliação e identificação de áreas e ações prioritárias para conservação, utilização sustentável e repartição dos benefícios da biodiversidade brasileira. MMA, SBF, Brasília.

CAMPANILI, M. \& SCHAFFER, W.B. 2010. Mata Atlântica: patrimônio nacional dos brasileiros. MMA, Brasília.

CHIARELlO, A.G., AGUIAR, L.M.S., CERQUEIRA, R., MELO, F.R., RODRIGUES, F.H.G. \& SILVA, V.M.F. 2008. Mamíferos ameaçados de extinção no Brasil. In Livro vermelho da fauna brasileira ameaçada de extinção (A.B.M. Machado, G.M. Drummond \& A.P. Paglia, eds.). MMA, Brasília, Fundação Biodiversitas, Belo Horizonte, v. 2, p.680-882.

COLWELL, R.K. EstimateS: statistical estimation of species richness and shared species from samples. versão 8.2. http://viceroy.eeb.uconn.edu/ EstimateSPages/EstimateS.flx (último acesso em 08/09/2011).

COSTA, L.P., LEITE, Y.L.R., MENDES, S.L. \& DITCHFIELD, A.D. 2005. Conservação de mamíferos no Brasil. Megadiversidade 1(1):103-112.

DANTAS, T.V.P., RIBEIRO, A.S., NASCIMENTO-JÚNIOR, J.E., MACHADO, A., CRISTINA, K. \& SANTOS, C.S. 2007. Caracterização florística. In Estudo técnico para a criação do Refúgio de Vida Silvestre da Mata do Junco, Capela, Sergipe (Sergipe. Secretaria de Estado do Meio Ambiente e dos Recursos Hídricos, org.). UFS, São Cristóvão, p.12-15.

ESBÉRARD, C.E.L. 2003. Diversidade de morcegos em área de Mata Atlântica regenerada no sudeste do Brasil. Rev. Bras. Zooc. 5(2):177-188. http://dx.doi.org/10.1590/S0101-81752005000200032

FARIA, D. 2006. Phyllostomid bats of a fragmented landscape in the NorthEastern Atlantic Forest, Brazil. J. Trop. Ecol. 22(5):531-542. http://dx.doi. org/10.1017/S0266467406003385

FARIA, D. \& BAUMGARTEN, J. 2007. Shade cacao plantations (Theobroma cacao) and bat conservation in Southern Bahia, Brazil. Biodivers. Conserv. 16:291-312. http://dx.doi.org/10.1007/s10531-005-8346-5

FARIA, D., SOARES-SANTOS, B. \& SAMPAIO, E. 2006. Bats from the Atlantic rainforest of Southern Bahia, Brazil. Biot. Neotrop. 6(2): http:// www.biotaneotropica.org.br/v6n2/pt/abstract?inventory+bn02406022006 (último acesso em 02/03/2011).

FEIJÓ, J.A. \& NUNES, H.L. 2010. Mammalia, Chiroptera, Phyllostomidae, Artibeus planirostris (Spix, 1823) and Trachops cirrhosus (Spix, 1823): first record for the state of Sergipe, Northeastern Brazil. Check List 6(1):15-16.
FERREIRA, R.A., ROCHA, J.C.S., SANTOS NETO, E.M., SANTOS, P.L. \& ABREU, L.D. 2007. Diagnóstico de nascentes. In Estudo técnico para a criação do Refúgio de Vida Silvestre da Mata do Junco, Capela, Sergipe (Sergipe. Secretaria de Estado do Meio Ambiente e dos Recursos Hídricos, org.). UFS, São Cristóvão, p.27-34.

FUNDAÇÃO SOS MATA ATLÂNTICA, INSTITUTO NACIONAL DE PESQUISAS ESPACIAIS - INPE. Atlas dos remanescentes florestais da Mata Atlântica: período 2008-2010. http://mapas.sosma.org.br/dados/ (último acesso em 18/06/2011).

GARDNER, A.L. 2008. Mammals of South America: marsupials, xenarthrans, shrews, and bats. University of Chicago Press, Chicago.

KUNZ, T.H. \& KURTA, A. 1988. Capture methods and holding devices. In Ecological and behavioral methods for the study of bats (T.H. Kunz, ed.). Smithsonian Institution Press, Washington, p.1-29.

LANDIM, M.F. \& SIQUEIRA, E.R. 2001. Caracterização florística e ecológica da Mata Atlântica de Sergipe. In Mata Atlântica de Sergipe (E.R. Siqueira \& F.E Ribeiro, eds.). Embrapa Tabuleiros Costeiros, Aracaju, p.9-50.

MIKALAUSKAS, J.S. 2005. Morcegos. In Parque Nacional Serra de Itabaiana: levantamento da Biota (C.M Carvalho \& J.C. Vilar, coords). IBAMA, Aracaju, UFS, São Cristovão, p.93-103.

MIKALAUSKAS, J.S., PERACCHI, A.L., GOUVEIA, S.F., ROCHA, P.A., VASCONCELOS, M.P.F. \& SILVEIRA, V.V. 2006a. Período de atividade de morcegos da família Phyllostomidae do Parque Nacional Serra de Itabaiana, Sergipe. Biol. Geral Exper. 6(2):11-13.

MIKALAUSKAS, J.S., MORATELLI, R. \& PERACCHI, A.L. 2006b. Ocorrência de Chiroderma doriae Thomas (Chiroptera, Phyllostomidae) no estado de Sergipe, Brasil. Rev. Bras. Zool. 23(3):877-878. http://dx.doi. org/10.1590/S0101-81752006000300035

MIKALAUSKAS, J.S., ROCHA, P.A., DIAS, D. \& PERACCHI, A.L. 2011. Mammalia, Chiroptera, Vespertilionidae Rhogeessa hussoni Genoways \& Baker, 1996: first record for the state of Sergipe, Northeastern Brazil. Check List 7(6):883-885.

MIRETZKI, M. \& MARGARIDO, T.C.C. 1999. Morcegos da Estação Ecológica do Caiuá, Paraná (sul do Brasil). Chirop. Neotrop. 5(1-2):105-108.

MITTERMEIER, R.A., GIL, P.R., HOFFMANN, M., PILGRIM, J., BROOKS, T., MITTERMEIER, C.G., LAMOUREX, J. \& FONSECA, G.A.B. 2005. Hotspots revisados: as regiões biologicalmente mais ricas e ameaçadas do planeta. Conservação Internacional, Brasil.

MULLER, M.F. \& REIS, N.R. 1992. Partição de de recursos alimentares entre quatro espécies de morcegos frugívoros (Chiroptera, Phyllostomidae). Rev. Bras. Zool. 9(3-4):345-355. http://dx.doi.org/10.1590/S010181751992000200022

ORTÊNCIO-FILHO, H. \& REIS, N.R. 2009. Species richness and abundance of bats in fragments of the stational semidecidual forest, Upper Paraná River, Southern Brazil. Braz. J. Biol. 69(2):727-734. http://dx.doi. org/10.1590/S1519-69842009000300026

PARDINI, R. 2004. Effects of forest fragmentation on small mammals in an Atlantic Forest landscape. Biodivers. Conserv. 13:2567-2586. http:// dx.doi.org/10.1023/B:BIOC.0000048452.18878.2d

PARDINI, R., FARIA, D., ACCACIO, G.M., LAPS, R.R., MARIANO-NETO, E., PACIENCIA, M.L.B., DIXO, M. \& BAUMGARTEN, J. 2009. The challenge of maintaining Atlantic Forest biodiversity: a multi-taxa conservation assessment of specialist and generalist species in an agroforestry mosaic in Southern Bahia. Biol. Conserv. 142(6):1178-1190. http://dx.doi.org/10.1016/j.biocon.2009.02.010

PARDINI, R., SOUZA, S.M., BRAGA-NETO, R. \& METZGER, J.P. 2005. The role of forest structure, fragment size and corridors in maintaining small mammal abundance and diversity in an Atlantic Forest landscape. Biol. Conserv. 124(2):253-266. http://dx.doi.org/10.1016/j. biocon.2005.01.033

PEDRO, W.A. \& TADDEI, V.A. 1998. Bats from Southwestern Minas Gerais, Brazil (Mammalia: Chiroptera). Chirop. Neotrop. 4(1):85-88. 
PERACCHI, A.L., GALLO, P.H., DIAS, D., LIMA, I.P. \& REIS, N.R. 2010 Ordem Chiroptera. In Mamíferos do Brasil: guia de identificação (N.R. Reis, A.L. Peracchi, M.N Fregonezi, B.K. Rossaneis, orgs.). Technical Books Editora, Rio de Janeiro, p.293-461.

PERACCHI, A.L., LIMA, I.P., REIS, N.R., NOGUEIRA, M.R. \& ORTÊNCIO-FILHO, H. 2011. Ordem Chiroptera. In Mamíferos do Brasil (N.R. Reis, A.L. Peracchi, W.A. Pedro \& I.P. Lima, eds.). 2. ed. N.R. Reis, Londrina, p.155-234.

REIS, N.R., BARBIERI, M.L.D.S., LIMA, I.P. \& PERACCHI, A.L. 2003. O que é melhor para manter a riqueza de espécies de morcegos (Mammalia, Chiroptera): um fragmento florestal grande ou vários fragmentos de pequeno tamanho? Rev. Bras. Zool. 20(2):225-230. http://dx.doi. org/10.1590/S0101-81752003000200009

REIS, N.R., PERACCHI, A.L., PEDRO, W.A. \& LIMA, I.P. 2007. Morcegos do Brasil. UEL, Londrina.

REIS, N.R., SHIBATTA, O.A., PERACCHI, A.L., PEDRO, W.A. \& LIMA, I.P. 2011. Sobre os mamíferos do Brasil. In Mamíferos do Brasil (N.R. Reis, A.L. Peracchi, W.A. Pedro \& I.P. Lima, eds.). 2. ed. N.R. Reis, Londrina, p.23-29.

REX, K., KELM, D.H., WIESNER, K., KUNZ, T.H. \& VOIGT, C.C. 2008. Species richness and structure of three Neotropical bat assemblages. Biol. J. Linn. Soc. 94:617-629.

RIBEIRO, M.C., METZGER, J.P., MARTENSEN, A.C., PONZONI, F.J. \& HIROTA, M.M. 2009. The Brazilian Atlantic Forest: How much is left, and how is the remaining forest distributed? Implications for conservation. Biol. Conserv. 142:1141-1153. http://dx.doi.org/10.1016/j. biocon.2009.02.021

ROCHA, P.A., FEIJÓ, J.A., MIKALAUSKAS, J.S. \& FERRARI, S.F. 2011 a. First records of mormoopid bats (Chiroptera, Mormoopidae) from the Brazilian Atlantic Forest. Mammalia 75:295-299. http://dx.doi. org $/ 10.1515 / \mathrm{mamm} .2011 .029$

ROCHA, P.A., FEIJÓ, J.A., MIKALAUSKAS, J.S., GOUVEIA, S.F., SILVEIRA, V.V. \& PERACCHI, A.L. 2010. Morcegos (Mammalia, Chiroptera) capturados no Campus da Universidade Federal de Sergipe, com oito novos registros para o estado. Biot. Neotrop. 10(3): http://www.biotaneotropica.org.br/v10n3/en/abstract?shortcommunication+bn03310032010. (último acesso em 02/03/2011).

ROCHA, P.A., FEIJÓ, J.A., RUIZ-ESPARZA, J. \& FERRARI, S.F. 2011 b. Uroderma magnirostrum Davis 1968 (Chiroptera, Phyllostomidae) First record from the state of Sergipe, Northeastern Brazil. Check List 7(6):886-888.
ROCHA, P.A., MIKALAUSKAS, J.S., SILVEIRA, V.V.B. \& MAGINA, G.C.T. 2007. Mamíferos. Diagnóstico de nascentes. In Estudo técnico para a criação do Refúgio de Vida Silvestre da Mata do Junco, Capela, Sergipe (Sergipe. Secretaria de Estado do Meio Ambiente e dos Recursos Hídricos, org.). UFS, São Cristóvão, p.22-23.

SANTOS, A.J. Estimativas de riqueza em espécies. 2003. In Métodos de estudos em Biologia da Conservação e Manejo da Vida Silvestre (L. Cullen Junior, R. Rudran \& C. Valladares-Pádua, orgs). Editora da UFPR, Fundação O Boticário de Proteção à Natureza, Curitiba, p.19-41.

SANTOS, L.V. \& SANTOS, M.J. 2006. Sergipe. In Mata Atlântica: uma rede pela floresta (M. Campanili \& M. Prochnow, orgs). RMA, Brasília, p.165-170.

SANTOS, M.J.S., SOUZA, H.T.R., SOUZA, R.M. 2007. Biomonitoramento através de indicadores ambientais abióticos - Mata do Junco (Capela-SE). Sci. Plena 3(5):142-151.

SCHULZE, M.D., SEAVY, N.E. \& WHITACRE, D.F. 2000. A comparison of the Phyllostomid bat assemblages in undisturbed Neotropical Forest and in forest fragments of a Slash-and-Burn farming mosaic in Petén, Guatemala. Biotropica 32(1):174-184. http://dx.doi.org/10.1111/j.1744-7429.2000. tb00459.x

SILVA, L.A.M., SOUZA, A.Q.S., LIMA, A.S., ARAÚJO, C.S.F., SILVA, C.V.M., SILVA, L.E.C., GOMES, M.F., QUEIROZ, P.L. \& SILVA, R.M. 2010. Morcegos da Reserva Particular do Patrimônio Natural Frei Caneca, nordeste do Brasil. Chirop. Neotrop. Supl. 16(1):87-90.

STRAUBE, F.C. \& BIANCONI, G.V. 2002. Sobre a grandeza e a unidade utilizada para estimar esforço de captura com utilização de redes de neblina. Chirop. Neotrop. 8(1-2):150-152.

TABARELLI, M., MELO, M.D.V.C. \& LIRA, O.C. A Mata Atlântica do Nordeste. http://www.amane.org.br/publicacoes.asp (último acesso em 14/06/2011).

TRAJANO, E. 1984. Ecologia de populações de morcegos cavernícolas em uma região cárstica do Sudeste do Brasil. Rev. Bras. Zool. 2(5):255-320. http://dx.doi.org/10.1590/S0101-81751984000100001

VIEIRA, M.V., FARIA, D.M., FERNANDEZ, F.A.S., FERRARI, S.F., FREITAS, S.R., GASPAR, D.A., MOURA, R.T., OLIFIERS, N., OLIVEIRA, P.P., PARDINI, R., PIRES, A.S., RAVETTA, A., MELLO, M.A.R., RUIZ, C.R. \& SETZ, E.Z.F. 2003. Mamíferos. In Fragmentação de Ecossistemas: causas, efeitos sobre a biodiversidade e recomendações de políticas públicas (D.M. Rambaldi \& D.A. Suárez, orgs.). MMA, SBF, Brasília, p.125-151.

VIZOTTO, L.D. \& TADDEI, V.A. 1973. Chave para determinação de quirópteros brasileiros. Bol. Ciências p. 1-72.

ZORTÉA, M. 2007. Subfamília Stenodermatinae. In Morcegos do Brasil (N.R. Reis, A.L. Peracchi, W.A. Pedro \& I.P. Lima, eds.). 2. ed. N.R. Reis, Londrina, p.107-128. 


\section{Apêndice}

Apêndice 1. Espécimes testemunhos depositados na Coleção de Mamíferos da Universidade Federal de Sergipe (CMUFS), Departamento de Biologia,

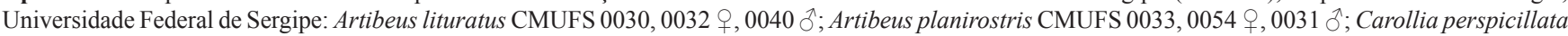

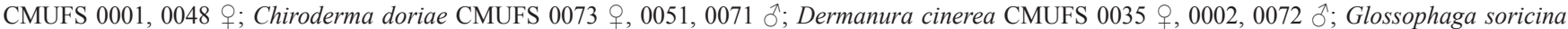
CMUFS 0038, 0068 \%; Myotis nigricans CMUFS 0036, 0037, 0039 ; Phyllostomus discolor CMUFS 0029, 0049, 0050 of, Platyrrhinus lineatus CMUFS

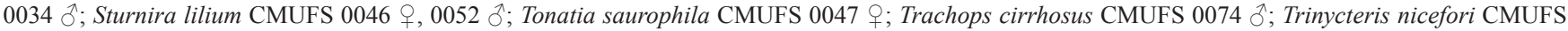
0055 ㅇ, 0056, 0063 ๙ .

Appendix 1. Vouchers deposited in the Coleção de Mamíferos da Universidade Federal de Sergipe (CMUFS), Departamento de Biologia, Universidade Federal

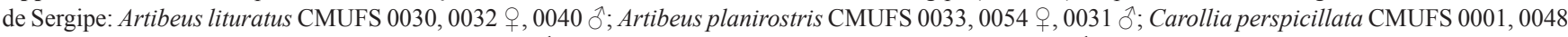

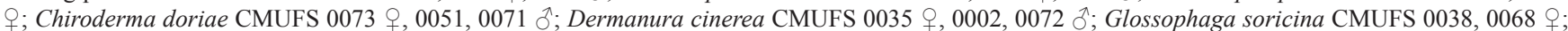

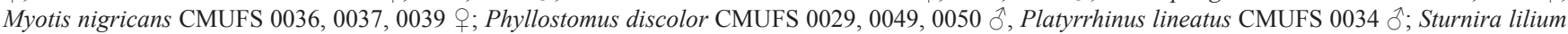

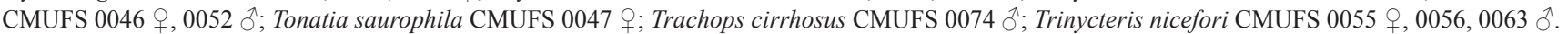

